\title{
Depressive Disorders Among Women Living in Resource Poor, Northern Kenya: The Role of Psycho-education
}

\author{
Gladys Kabura Mwangi \\ Africa International University, Nairobi, Kenya
}

\begin{abstract}
This paper highlights the findings of a dissertation research which aimed to determine the effectiveness of a model of psycho-education (PE) developed for this study, in treating the symptoms of depressive disorders among Maasai women living in resource poor settings in Laikipia County, in Northern Kenya. The quasi-experimental research design with an experimental group (EG) and control group (CG) was adopted for this study. The target population for this study were 686 female members of Conservation Enterprise Groups (CEG) operating in the area. A sample size of 200 was selected for the study; (EG, $\mathrm{n}=100)$ and $(C G, \mathrm{n}=100)$, at $80 \%$ power and $30 \%$ effective size. The tool used to test for symptoms of depression was the Beck's Depression Inventory (BDI). The PE was provided as an intervention treatment to the EG at the middle and end of the study, after which the respondents were tested for symptoms of depression. The prevalence of depression was established at $26.7 \%$ and statistical tests conducted throughout the studies showed that the $\mathrm{PE}$ treatment significantly improved depression symptoms $(\mathrm{P}<0.005)$. Data from the $(C G)$ showed that if these symptoms were not treated using, they degenerated into severe symptoms. Data was analysed using SPSS Version 21.0.
\end{abstract}

Keywords: psycho-education treatment for depressive disorders, Maasai women, Northern Kenya

\section{Background \& Introduction to the Study}

Mental Health Disorders are prevalent in Africa. In South Africa, a study that looked at factors associated to 12-month and lifetime prevalence of common mental disorders indicated that the lifetime prevalence for any disorder was 30.3\% (Herman, Stein, Seedat, Heeringa, Moomal, \& Williams, 2009). Further, the study indicated that the most prevalent 12-month and lifetime disorders were those related to anxiety disorders.

In Kenya, a study conducted in primary health care settings to estimate the prevalence of common mental disorders, established that major depression is a common mental health disorder among adults in the region in Western Kenya with a prevalence of 26.3\% (Allion, Ndetei, Khasakhala, Ngari, Achola, Akinyi, \& Ribero, 2014). Studies conducted in Kenya, showed that the prevalence of common mental health disorders in resource poor settings is $10.8 \%$ (Jenkins et al., 2012); further depressive symptoms are rampant among women in resource poor settings in Northern Kenya, with a prevalence of 26.7\% (Mwangi, 2017). This evidence connotes that major depressive disorders are rampant in Kenya and that there is need for appropriate interventions to screen for, identify, and treat the same while giving specific consideration to those at risk of contracting the same.

Gladys Kabura Mwangi, Dr.,/Ph.D., Clinical Psychology, Department of Counselling Psychology, Africa International University, Nairobi, Kenya. 
Psycho-education (PE) could play an essential role in rehabilitating, re-establishing, and treating people with anxiety disorders, improving their overall well-being (Bartells, 2004). It refers to the interventions that are offered to people or groups with mental health conditions including depressive disorders, to impart therapeutic, cognitive, and social benefits. To achieve this, PE uses education, goal setting, skill teaching, challenging thinking patterns, and enhancing social interaction (Morse, 2004). In this study, the same definition was used to guide the eclectic model of psycho-education that was developed and administered to women with depressive symptoms.

Research has shown that most PE interventions are not preceded by psychological assessments. Further, the mental or psychological needs that they are intended to address are often not diagnosed in line with the previous Diagnostic \& Statistical Manual of Mental Disorders (DSM-IV) (Bartells, 2004) and DSM-V (Mwangi, 2017) criterion. Further, they are mostly implemented based on assumptions of the symptoms that are observed (not tested) at first presentation (Bartells, 2004). In this regard, it appears as though only a few of $\mathrm{PE}$ content that are implemented in resource poor settings meet the precise psychological needs of communities or groups, for which they are tailor made.

Previously there was a general belief that PE interventions are unproductive and inert. However, a global meta-analysis of literature on psycho-education interventions showed that the ones for psychological distress (including depression) could positively symptoms (Donker, Griffiths, Cuijpers, \& Christensen, 2009). However, the authors noted that the quality of the content of psycho-education is critical. However, it needs to be matched to the needs of individuals and groups in the community. Further psychological assessments need to form the basis of all PE programs (Mwangi, 2017).

This study aimed to examine the effectiveness of an eclectic model of psycho-education (developed for this study) in treating depressive symptoms amongst Maasai women living in resource poor settings in Laikipia County in Northern Kenya. The Beck's Depression Inventory (BDI) tool was used to test for depressive symptoms among the participants of the study. The PE treatment was administered to respondents in the experimental group (EG) $(n=107)$ and not provided to the control group $(C G)(n=101)$, during the first (midline) and second endline interventions.

However, due to ethical considerations, the intervention was provided to the CG at the end of the study after the data collection process was complete. This was done to mitigate the escalating symptoms of depressive disorders among the respondents in the CG. Time series tests conducted to assess the overall means of anxiety after the psycho-education treatment indicated a significant reduction of depressive symptoms in the EG.

The knowledge and evidence for treating depressive disorders using PE in resource poor settings that was generated by this study could be useful to policy makers and development practitioners, pursuing collection of indicators and data on mental health indicators that needed to inform SDG (3), which focuses on "ensuring healthy lives and promoting well-being for all...".

This study was also critical because it also provided evidence which could help to integrate and improve mental health well-being of people living in resource poor settings. Further, findings of this study could contribute to the mental health arena by providing a framework for assessing psychological disorders such as depression in resource poor settings in Kenya and the minimum package of PE developed for treating depressive symptoms could be replicated for women living in other resource poor settings in the region and globally. 


\section{Study Methods}

The quasi-experimental research design was adopted for this study. The population for this study was 686 female members of Conservation Enterprise Groups (CEG) in Laikipia North. A sample size of 200 for both the experimental (EG, $n=100)$ and control group $(C G, n=100)$ was selected at $80 \%$ power and $30 \%$ effective size (Chan, 2003). A study conducted in Kenya showed that PE had a 30\% effect on subjects with depression and a $29 \%$ effect on subjects with anxiety (Muriungi \& Ndetei, 2013). In relation to this study, the PE intervention was anticipated to have a successful and clinical relevance of $30 \%$ (effect size) translating to $70 \%$ of the subjects participating in the study (Chan, 2003). This rationale informed the sample size selection.

The Beck's Depression Inventory (BDI), a standardised tool that is used for testing for depression was used to collect data on depression. The inclusion criteria for those selected to participate in the study included those who tested positive for mild to moderate scores for depression (14 to 29), as tested by the BDI. Those with minimal and severe depression scores $(14>x>29)$ were not eligible to participate in the study. However, as part of the ethical requirements, the researcher referred all the respondents in the EG who presented with severe depression symptoms to the Nanyuki Teaching and Referral Hospital for clinical treatment. This inclusion and exclusion criteria was used to identify participants for the $\mathrm{CG}$ as well and the ethical principle for those found with severe depression was also applied for the same.

107 female participants who met the criteria for minimal to moderate depressive symptoms were recruited to participate in the treatment intervention for the experimental group (EG) and 101 women were recruited to participate in the control group (CG). These women either were active members of or were affiliated to CEGs. The reason why more respondents were recruited to participate in the study beyond the specified sample size is because, after the announcement about the study was made, women turned up in large numbers because they felt that depressive disorders were critical and affected many women in the community.

Further to this, some of the women had travelled long distances to participate in the study and the researcher felt obliged to include them in the study. The PE treatment was only administered to the EG. Data was analysed and significance tests conducted using SPSS Version 21.0 and presented in tables and graphs.

\section{Research Objective, Question, and Hypothesis}

One of the objectives of the study was to determine the effectiveness of psycho-education in improving depressive disorders.

Other specific objectives related to this study were as follows:

- To establish the prevalence of prevalence of depressive disorders among women in the CEGs.

- To determine the effect of psycho-education in treating depressive disorders.

\section{Research Question}

Does psycho-education have an impact on depressive symptoms?

\section{Null Hypothesis}

Psycho-education does not an impact on depressive symptoms.

\section{Results}

\section{Prevalence of Mild Depression}

The study established that the prevalence of mild depression in the EG was as $14 \%$ as compared to $26.7 \%$ in the CG at baseline. This prevalence reduced to $0 \%$ in the EG after the first PE treatment for EG, but also 
reduced to and $15.8 \%$ for the CG. However, after the second and final treatment the prevalence for mild depressive symptoms had increased to $1.9 \%$ for EG but had increased to $21.8 \%$ in the CG. The drop in prevalence of mild depression in the EG after the first treatment could be because of more respondents testing positive for moderate depression as indicated by the prevalence for the same at the second treatment (See Table 1).

\section{Prevalence of Moderate Depression Symptoms}

At baseline, findings of the study indicated that the prevalence of moderate depression in the EG was $14 \%$ as compared to $26.7 \%$ in the CG. However, after the first PE intervention and treatment, the prevalence reduced to $0 \%$ in the EG and reduced to $16.8 \%$ in the CG. Further, after the second and final PE treatment, no respondent in the EG had moderate depression in EG, but the prevalence rose to $27.7 \%$ in the CG (See Table 1). The drop in prevalence of mild depression in CG after the first treatment could be due to more respondents testing positive for moderate symptoms of depression at the same timeline. However, an increase in prevalence could indicate that the respondents were more aware of the symptoms of the disorders that they psychologically started experiencing the same. This was corroborated with data from increased hospital visits.

Table 1

Prevalence of Depressive Symptoms for Both EG and CG

\begin{tabular}{|c|c|c|c|c|c|c|c|c|c|c|c|c|}
\hline & \multicolumn{4}{|c|}{ Baseline } & \multicolumn{4}{|c|}{ Midline } & \multicolumn{4}{|c|}{ Endline } \\
\hline & \multicolumn{2}{|c|}{$\mathrm{N}=107$} & \multicolumn{2}{|r|}{$\mathrm{N}=101$} & \multicolumn{2}{|r|}{$\mathrm{N}=105$} & \multicolumn{2}{|c|}{$\mathrm{N}=101$} & \multicolumn{2}{|r|}{$\mathrm{N}=105$} & \multicolumn{2}{|c|}{$\mathrm{N}=101$} \\
\hline & & $G$ & & CG & & EG & & $\mathrm{CG}$ & & EG & & $\mathrm{CG}$ \\
\hline & $\mathrm{n}$ & $\%$ & $\mathrm{n}$ & $\%$ & $\mathrm{n}$ & $\%$ & $\mathrm{n}$ & $\%$ & $\mathrm{n}$ & $\%$ & $\mathrm{n}$ & $\%$ \\
\hline Prevalence mild depression & 24 & 22.4 & 21 & 20.8 & 0 & 0 & 16 & 15.8 & 2 & 1.9 & 22 & 21.8 \\
\hline $\begin{array}{l}\text { Prevalence moderate } \\
\text { Depression }\end{array}$ & 15 & 14.0 & 27 & 26.7 & 0 & 0 & 17 & 16.8 & 0 & 0 & 28 & 27.7 \\
\hline Prevalence severe depression & 0 & 0 & 0 & 0 & 0 & 0 & 0 & 0 & 0 & 0 & 15 & 14.9 \\
\hline
\end{tabular}

Time series tests established that the PE treatment effectively significantly reduced the prevalence of depressive symptoms. A time series analysis using mean scores for both the EG and CG groups showed that depression scores fell from a mean score of 1.74 at the baseline to 1.50 after the first psycho-education treatment but rose again to a mean score of 2.22 after the second psycho-education treatment for CG (See Table 2).

The drop in the mean scores at midline could have been because of the respondents becoming more aware of the symptoms of depression as an illness and not a norm. In the EG there was a decrease in mean scores for depressive symptoms from baseline to the final PE treatment, from 1.50 at baseline to 1.00 after the first psycho-education treatment (midline) and subsequently to 1.02 after the final psycho-education treatment intervention at endline (See Table 2).

Table 2

Time Series Testing the Overall Means of Anxiety and Depression After Psycho-education Treatment

\begin{tabular}{lllllll}
\hline & \multicolumn{3}{c}{ Baseline } & \multicolumn{2}{c}{ Midline } & \multicolumn{2}{c}{ Endline } \\
\cline { 2 - 7 } & EG & CG & EG & CG & EG & CG \\
\hline Depression scores & 1.50 & 1.74 & 1.00 & 1.50 & 1.02 & 2.22 \\
\hline
\end{tabular}




\section{Effects of Psycho-education on Depressive Disorders}

To ascertain the impact of the PE on depression, all the data for baseline, midline, and end line were combined to achieve a continuous data for both EG \& CG. This was useful in helping to conduct T-tests on independent samples. This test was then used to compare the means between the EG and CG at each level of assessment, such as (i) at baseline, (ii) at treatment/Intervention 1 (midline), and (iii) at treatment/Intervention 2 (endline). These tests indicated that the PE treatment significantly reduced depressive symptoms among the women (See Table 3).

Table 3

T-test After Psycho-education Treatment/Intervention 2

\begin{tabular}{|c|c|c|c|c|c|c|c|c|}
\hline & & \multicolumn{7}{|c|}{ T-test for equality of means } \\
\hline & & \multirow[t]{2}{*}{$\mathrm{t}$} & \multirow[t]{2}{*}{ df } & \multirow{2}{*}{ P-value } & \multirow{2}{*}{$\begin{array}{l}\text { Mean } \\
\text { difference }\end{array}$} & \multirow{2}{*}{$\begin{array}{l}\text { Std. error } \\
\text { difference }\end{array}$} & \multicolumn{2}{|c|}{$\begin{array}{c}95 \% \text { confidence interval of } \\
\text { the difference }\end{array}$} \\
\hline & & & & & & & Lower & Upper \\
\hline \multirow{2}{*}{$\begin{array}{l}\text { Depression } \\
\text { scores }\end{array}$} & $\begin{array}{l}\text { Equal variances } \\
\text { assumed }\end{array}$ & 10.711 & 196 & 0.000 & 1.197 & 0.112 & 0.977 & 1.418 \\
\hline & $\begin{array}{l}\text { Equal variances not } \\
\text { assumed }\end{array}$ & 10.923 & 103.562 & 0.000 & 1.197 & 0.110 & 0.980 & 1.415 \\
\hline
\end{tabular}

\section{ANOVA Tests for Control Group}

The test for homogeneity of variances indicated depressive symptoms scores of $\mathrm{P}<0.05$. This indicates that there was a statistically significant difference in depressive symptoms for the CG, which seemed to worsen among the women during the three timelines (See Table 4). This finding led to the conclusion that due to the absence of the PE treatment in the CG, the symptoms became worse among the respondents.

Table 4

ANOVA Tests to Measure Depressive Symptoms in the Absence of the PE Treatment for the Control Group

\begin{tabular}{lllll}
\hline & Levene statistic & df1 & df2 & P-value \\
\hline Depression scores & 13.080 & 2 & 300 & 0.000 \\
\hline
\end{tabular}

\section{ANOVA Scores for Experimental Group}

ANOVA tests performed for depressive symptoms among respondents in the EG after the final PE treatment indicated p-value of $\mathrm{P}<0.05$ (See Table 5). This showed that the treatment had resulted to a significant reduction in the symptoms.

Table 5

ANOVA Tests to Measure Depressive and Anxiety Symptoms After the PE Treatment in the Experimental Group

\begin{tabular}{lllll}
\hline & Levene statistic & df & df2 & P-value. \\
\hline Depression scores & 260.901 & 2 & 298 & 0.000 \\
\hline
\end{tabular}

\section{Discussion}

Findings of the T-tests and ANOVA tests conducted indicated that the psycho-education treatment led to a significant reduction in depressive symptoms among the women in the EG. These findings are similar to findings conducted by Xavier et al. (2010) in South Africa, Donker et al. (2009) in the USA, Dyck, Hendryx, Short, Voss, and McFarlane (2002) in Britain, and Muriungi and Ndetei (2013) and Kyobutungi, Egondi, and 
Ezeh (2010) in Kenya, which indicated that psycho-education has a positive impact on improving anxiety and depressive symptoms leading to improvements in QoL.

This evidence showed that the PE treatment administered to respondents in the study significantly reduced depressive symptoms. This finding is contrary to those of another meta-analysis of PE interventions, which showed that the intervention had a small but significant effect on depression (Donker et al., 2009). Therefore based on this finding, the psycho-education treatment model adopted for this study was effective in treating the depressive symptoms among women in the EG.

\section{Summary of Key Findings}

Depressive symptoms are prevalent among Maasai women in Northern Kenya. Qualitative data collected during the focus group discussions (FGDs); the women identified with and defined symptoms of depression as a condition they called in their local language as "Aisinanu". This enabled them to narrate and give feedback of how they experienced its symptoms and how it impacted on their day to day lives livelihoods. The study established that other socio-cultural and environmental factors such as being in polygamous relationships helped to reduce depression, and particularly among the older women. This led to conclude that some older women in the Masaai community view polygamy as a positive cultural practice, which helps to mitigate depressive symptoms.

\section{Conclusions}

The study found out that depressive symptoms are prevalent among Maasai women in Laikipia County in Northern Kenya. Further it determined that the PE treatment developed and provided during the intervention significantly reduced depressive symptoms amongst the women in EG. This creates a need to identify low-cost measures to respond to mental health needs of women with anxiety and depressive disorders.

Such low cost measures could include PE approaches that could be taught and administered by trained community members in local settings that are resource poor. These interventions could then be linked and integrated to mental health services in primary healthcare settings. Such an approach could help to ensure access to mental health services including depressive symptoms for women and members of communities living in resource poor settings.

Further, the study also established that other socio-cultural factors such as being in a polygamous marriage - where the husband had many wives, having many children, absence of conflict over scarce water resources between humans and wildlife and lack domestic violence, were all viewed as important in mitigating depressive symptoms among the women female respondents.

\section{Recommendations}

Due to the high prevalence of depressive symptoms among women in resource poor settings in Laikipia, the study recommended that there is need for national and international mental health strategies, to integrate psychological assessments such as the BDI, to evaluate mental health issues including depressive symptoms. This would help to ascertain the psychological needs for which cost-effective PE interventions will be tailor made.

Few studies exist on PE programs that address the mental health needs of women including young women with anxiety symptoms in resource poor settings, in Northern Kenya. In this regard the study therefore 
recommended that global and regional organizations, as well as academic institutions could in future adopt and or improve on the PE model that was used in this study. This could help to improve mental health outcomes of women (and especially younger ones), in resource poor settings and further improve their socio-economic and political livelihoods. However, to ensure support within their homes, it is critical to involve the women's spouses (for the married women) in the interventions, so as to enhance their social, economic, and political decision-making and bargaining power at home.

\section{Recommendations for Further Research}

This study recommended the following for further research:

The study recognized the need to further investigate other socio-economic and cultural factors that could cause depression disorders among women in resource poor settings. Further, there is need for further research on traditional and cultural approaches for treating depressive symptoms, which could be integrated in PE interventions in resource poor settings. Such research could also help to mitigate and prevent incidences and the spread of anxiety.

\section{References}

Aillon, J. L., Ndetei, D. M., Khasakhala, L., Ngari, W. N., Achola, H. O., Akinyi, S., \& Ribero, S. (2014). Prevalence, types and comorbidity of mental disorders in a Kenyan primary health centre. Social Psychiatry of Epidemiol, 49(8), 1257-1268.

Bartels, S. J. (2004). Caring for the whole person: Integrated health care for older adults with severe mental illness and medical comorbidity. Journal of the American Geriatrics Society, 52(12), 249-257.

Chan, H. Y. (2003). Randomised controlled trials (RCTs)-sample size: The magic number? Singapore Medical Journal, 44(4), $172-174$

Christensen, H., Griffiths, K. M., \& Jorm, A. F. (2004). Delivering interventions for depression by using the internet: Randomised controlled trial. British Medical Journal, 328, 265-268.

Donker, T., Griffiths, K. M., Cuijpers, P., \& Christensen, H. (2009). Psychoeducation for depression, anxiety and psychological distress: A meta-analysis. BMC Medicine, 16(7), 79-89.

Dyck, G. D., Hendryx, S. M., Short, A. R., Voss, D. W., \& McFarlane, R. W. (2002). Service use among patients with schizophrenia in psychoeducational multiple family group treatment. Psychiatric Services, 53(6), 749-754.

Herman, A. A., Stein, D. J., Seedat, S., Heeringa, S. G., Moomal, H., \& Williams, D. R. (2009). The South African stress and health (SASH) study: 12-month and lifetime prevalence of common mental disorders. South African Medical Journal, 99(2), 339-344.

Jenkins, R., Njenga, F., Okonji, M., Kigamwa, P., Baraza, M., Ayuyo, J., ... \& Kiima, D. (2012). Prevalence of common mental disorders in a rural district of Kenya, and socio-demographic risk factors. International Journal of Environmental Research and Public Health, 9(5), 1810-1819.

Kyobutungi, C., Egondi, T., \& Ezeh, A. (2010). The health and well-being of older people in Nairobi's slums. Australia: World Health Organization.

Morse, W. C. (2004). Psychoeducational perspective overview. Michigan: University of Michigan.

Muriungi, S., \& Ndetei, D. M. (2013). Effectiveness of psycho-education on depression, hopelessness, suicidality, anxiety and substance use among basic diploma students at Kenya Medical Training College. South African Journal of Psychology, 19(2), 41-50.

Mwangi, G. K. (2017). The effect of psycho-education on the quality of life of women with comorbid symptoms of depression and anxiety in resource poor settings. African Journal of Clinical Psychology, School of Human \& Social Sciences, 1(1), 36-50. Daystar University, 44400-00100.

Ndetei, A., Khasakhala, L., Mbwayo, A., \& Mutiso, V. (2011). Epidemiological patterns of anxiety disorders in Kenya. Kenya: Africa Mental Health Foundation (AMHF). Retrieved from www.intechopen.com

Xavier, F., Gomez,O., Thorogood, M., Clark, B., D., Kahn, Kathleen., \& Tollman, S., M. (2010) . Assessing health and well-being among older people in rural South Africa. Global Health Action. South Africa. 